\title{
Model-Based Estimation of Thin Multi-Layered Media Using Ultrasonic Measurements
}

\author{
Fredrik Hägglund, Jesper Martinsson, and Johan E. Carlson
}

\begin{abstract}
In ultrasonic measurement situations, when dealing with media of multi-layered structures consisting of 1 or more thin layers, analysis of the measured ultrasonic waveform can be difficult because of overlapping and reverberant echoes. Information from the individual layers is then difficult to extract because the individual echoes cannot be detected. In this study, we use a parametric layer model to analyze the multi-layered material in a system identification approach. The parameters of the model are connected to physical properties of the investigated material, e.g., the reflection coefficients, the time-of-flight, and the attenuation. The main advantage using this model is that the complexity of the model is connected to the number of layers rather than the number of observable echoes in the received ultrasonic waveform. A system of linear equations is presented, giving the opportunity to find the model for both pulse-echo and through-transmission measurements. A thorough effort is made on the parameter estimation and optimization algorithm. The model is validated with practical measurements on a 3-layered structure using both pulse-echo and through-transmission techniques. The 3-layered material consists of a thin embedded middle layer with the time-of-flight in that layer shorter than the emitted signal's time support, giving rise to overlapping echoes. Finally the relation between the model parameters and physical properties of the material is established.
\end{abstract}

\section{INTRODUCTION}

$\mathrm{I}_{\mathrm{t}}^{\mathrm{N}}$ $\mathrm{N}$ ultrasonic measurements using pulse-echo or throughtransmission configurations, the received ultrasonic echoes are evaluated to retrieve information about the inspected media. Depending on the media under inspection, the extractable information from the received echoes varies. Characterizing the propagation path can give information on the media and, if possible, scatterers present (particles in a gas or a fluid, a solid medium, a grain or a defect in a solid medium, etc.). Inspecting the propagation path can give information about the size, location, and orientation of these scatterers. Finding information about the physical properties of the propagation path or the scatterers can be performed by examining the signal waveform. This approach is appropriate for time-delay estimation when we have non-overlapping echoes and rela-

Manuscript received September 18, 2008; accepted April 26, 2009.

F. Hägglund is with EISLAB, Department of Computer Science and Electrical Engineering, Luleå University of Technology, Luleå, Sweden (e-mail: Fredrik.Hagglund@ltu.se).

J. Martinsson is with LKAB R\&D, Malmberget, Sweden.

J. E. Carlson is with the Division of Systems and Interaction, Department of Computer Science and Electrical Engineering, Luleå University of Technology, Luleå, Sweden.

Digital Object Identifier 10.1109/TUFFC.2009.1233 tively high signal-to-noise ratio [1]. For other situations and when estimating other properties, this method has limited use. Another approach is to use a model, either a non-parametric model (the same number of parameters as data points) or a parametric model. Several different non-parametric and parametric techniques have been used to model materials and structures [2]-[8]. These types of models usually have limited use when dealing with overlapping echoes and in situations with low SNR. Parametric models have advantages in situations with low SNR, often avoiding undesired biased estimation result [9]. The main advantages with the parametric model proposed in this paper are that it can handle overlapping situations, the complexity is connected to the number of layers, and the estimated parameters are directly connected to the properties of the investigated media.

Finding the desired information in an ultrasonic waveform requires appropriate parametric models of either the formation of the received echoes, see [10]-[12], or of the inspected media. In this study, the focus is on modeling the media under investigation with a parametric model, estimation of the parameters of that model, and on the analysis of the model parameters. The study examines the case of normal incidence ultrasonic waves, and the media under investigation are materials with multi-layered structures consisting of 1 or more thin layers. Opposite from modeling the individual echoes in the waveform [11], [12], the layered model is valid for any kind of signal waveform and not depending on the excited signal's appearance. Dealing with thin layers (the time-of-flight through the layer is smaller than the signals time support) imply a received waveform consisting of several reverberant and overlapping echoes. A parametrization of the layered structure instead of each echo is then preferable, since the total number of parameters in the model can be kept small (independent of the number of observable echoes), and are related to physical properties of the investigated materials.

The thickness of the thin embedded middle layer in the experimental study is in the order of the wavelength of the ultrasonic wave in that layer, creating a response signal consisting of overlapping waveforms. Increasing the transducer frequency and decreasing the pulse width is often the usual approach to prevent overlaps. However, there are several situations when this approach is not an option (e.g., too short propagation distance, high-frequency attenuation and absorption, or hardware limitations). In these situations, the model described in this paper is 
very useful. Note that the model is also applicable for the completely separated case, changing the focus from interpreting and comparing different waveforms to modeling the layered media.

From the estimated model, it is possible to get an estimation of the difference in time of the echoes, such as the time-of-arrival (TOA), time-of-flight (TOF), or time difference of arrival. The estimated time delay can be used to estimate distance or sound velocity assuming that the other is known. The distance measurement can be a thickness, a location, a size, etc., and it is easier to interpret when the propagation path is homogenous. TOF is also used for surface profiling [13], where the knowledge of the surroundings, the experimental setup, is important.

Non-destructive testing (NDT) of materials using ultrasound has a wide range of applications in the area of process control, see for example [14]-[16]. Relative changes in the estimated parameters of the received echoes can be used to monitor changes in the material that can be due to fluctuations in the process. NDT of materials using ultrasound is also considered a valuable tool to characterize thin layers in a multi-layered material. As mentioned previously, several difficulties arise when dealing with thin layers. In this study, we use a parametric layer model to analyze the multi-layered material using a system identification approach, where the parameters of the model are associated with physical properties of each layer within the structure.

The paper is organized as follows. In Section II, a theoretical background is given to the wave propagation, reflection and transmission coefficient, and acoustic properties of the layer. The propagation path is rewritten using linear system notations, input and output signals are defined, and different measurement setups are discussed. In Section III, the complete layer model is presented, starting with describing a single layer within a multi-layered structure. Then the 1-, and 2-layered models are derived to exemplify the model structure, and finally the $Q$-layered model is given. A description of the parametrization and the model validation is also given. In Section IV, the maximum likelihood estimator of the model parameters is derived and the optimization algorithm is given. In Section $\mathrm{V}$, the experimental setup is explained, and in Section VI, estimation results from practical measurements are presented to validate the model.

\section{Theoretical BACKGRound}

This section begins by stating the wave equation for a lossy medium. Then an explanation of how the acoustic pressure is refracted and transmitted at the boundary between 2 media is given, by stating the reflection and transmission coefficients. Furthermore, the solution to the 1-way wave equation is examined, resulting in an explanation of the time-of-flight and the attenuation. Finally, the linear system is defined and issues concerning the input signal are discussed.

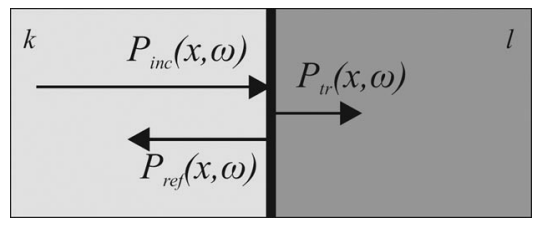

Fig. 1. An incident wave, $P_{\text {inc }}(x, \omega)$, is refracted into a reflected wave, $P_{\text {ref }}(x, \omega)$, and a transmitted wave, $P_{\operatorname{tr}}(x, \omega)$, at the boundary location $x$, between the $k$ th and $l$ th medium.

\section{A. Wave Equation}

A correctly designed experimental setup and a material with layers oriented in parallel will verify the assumption of plane waves and perpendicular incident angles, implying that the effect of mode conversion will decrease and only longitudinal waves are present [17]. Furthermore, it is assumed that linear acoustics apply, that is, the linear lossy wave equation, in the 1-D space, can be expressed as [18]

$$
\frac{\partial^{2}}{\partial x^{2}} P(x, \omega)=-k^{2}(\omega) P(x, \omega)
$$

where $x$ is the spatial variable, $\omega$ is the angular frequency, $P(x, \omega)$ is the Fourier transform of the acoustic pressure, and $k(\omega)$ is the complex frequency dependent wave number.

\section{B. Reflection and Transmission Coefficient}

A medium has a descriptive property in the acoustic impedance. For a low-loss medium the acoustic impedance, measured in $\mathrm{Pa} \cdot \mathrm{s} / \mathrm{m}$, can be written as $z=\rho c$, where $\rho$ is the density $\left(\mathrm{kg} / \mathrm{m}^{3}\right)$ and $c$ is the speed of sound $(\mathrm{m} / \mathrm{s})$. The acoustic impedance is the important factor when describing the reflected and transmitted part of an incident wave at a boundary. At the boundary the continuity of force and velocity is applied and the incident wave is divided into a reflected and a transmitted wave [18], see Fig. 1.

Assuming the incident wave to be perpendicular to the surface, the reflected and the transmitted waves are connected to the incident wave as

$$
\begin{gathered}
P_{\text {ref }}(x, \omega)=R_{k, l} P_{\text {inc }}(x, \omega), \\
P_{\text {tr }}(x, \omega)=T_{k, l} P_{\text {inc }}(x, \omega),
\end{gathered}
$$

where $R_{k, l}=\left(z_{l}-z_{k}\right) /\left(z_{k}+z_{l}\right)$ is the reflection coefficient and $T_{k, l}=2 z_{l} /\left(z_{k}+z_{l}\right)$ is the transmission coefficient and the subscripts denote the 2 media. An oblique incident wave will require inclusion of the angle of incidence; however, in this study we assume incident waves perpendicular to the surface.

\section{Acoustic Properties Inside a Layer}

The solution to (1) in the positive $x$-direction is called the 1-way wave equation [19] and is given by 


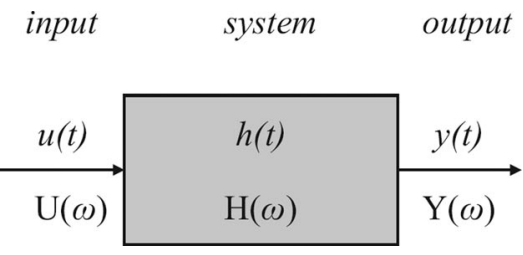

Fig. 2. A linear system connecting the input signal to the output signal. The notations are in both the time and the frequency domains.

$$
\begin{gathered}
P(x, \omega)=P\left(x_{0}, \omega\right) e^{-j k(\omega)\left(x-x_{0}\right)}, \\
=P\left(x_{0}, \omega\right) e^{-j\left(\omega /\left(c_{p}(\omega)\right)\right)\left(x-x_{0}\right)-\alpha(\omega)\left(x-x_{0}\right)},
\end{gathered}
$$

where $j=\sqrt{-1}$, and $P\left(x_{0}, \omega\right)$ is the pressure at the initial location $x_{0}$. The complex frequency dependent wave number is defined as $k(\omega)=\omega / c_{p}(\omega)-j \alpha(\omega)$, where $c_{p}(\omega)$ is the frequency dependent phase velocity of the wave, and $\alpha(\omega)$ is the frequency dependent attenuation coefficient, expressed in nepers per meter. The first part of the exponent is connected to the time-of-flight (phase delay) and the second part to the attenuation of the wave. The rate at which the amplitude of the wave is decreasing depends on the medium and the frequency of the wave. The attenuation can be described as the pressure change of a wave due to absorption, scattering, and diffraction. In classical absorption theory, the attenuation coefficient can be approximated to be proportional to the square of the sound frequency, $\alpha(\omega)=\alpha \omega^{2}$, which is a novel and appropriate model that captures the main characteristics of the observable attenuation.

Rewriting the relative distance traveled by the wave as $d=x-x_{0}$ and defining the time-of-flight (constant phase delay) for a constant phase velocity as $\tau=\left(x-x_{0}\right) / c_{p}$, we can model the collected exponential terms inside the qth layer as

$$
M_{q}(\omega)=e^{-j \omega \tau_{q}-\alpha_{q} \omega^{2} d_{q}}
$$

where $\tau_{q}$ is the time-of-flight and $\alpha_{q}$ is the attenuation coefficient in layer $q$, and $d_{q}$ is the thickness of layer $q$.

\section{Linear System}

Using a system identification approach, the ultrasonic measurement system can be considered as a linear system if linear acoustics apply. The material or specimen under investigation is considered as the transfer function, connecting an input signal (reference) to an output signal (response), see (7) and Fig. 2. The emitted signal from the transducer is the input signal to the system and the received echoes forming the ultrasonic waveform is the output of the system. The emitted signal from the transducer is unknown and the input to the system needs to be estimated.

If the assumption of linear acoustics holds, there exists a linear system with impulse response $h(t)$ that describes the relationship between the output signal $y(t)$ and the input signal $u(t)$. The main part of the analysis in this study is performed in the discrete frequency domain, resulting in several advantages compared with time-domain techniques [20]. The main advantage in this study is that operations using derivatives, e.g., to find critical points, are easily implemented in the frequency domain (avoiding discrete difference approximations).

The equation that describes how the transfer function $\mathrm{H}(\omega)$ relates the output signal $\mathrm{Y}(\omega)$ and the input signal $\mathrm{U}(\omega)$ is then defined as

$$
\mathrm{Y}(\omega)=\mathrm{H}(\omega) \mathrm{U}(\omega)
$$

where the capital letters denote Fourier-transformed quantities. In the simplest case, considering a wave traveling in a homogenous medium without boundaries, the transfer function is defined as

$$
\mathrm{H}(\omega)=M_{q}(\omega)
$$

where $M_{q}(\omega)$ is the model for the material properties inside the qth layer, defined in (6).

\section{E. Input Signal}

The estimation of the input signal is highly dependent on the measurement configuration (pulse-echo or throughtransmission), frequency of the transducer, and the specimen under investigation. In a pulse-echo configuration it is possible to estimate the input signal from the measured signal waveform. This requires separable echoes because both the input and the output signal needs to be extracted from the same measured signal waveform. Under the assumption of linear acoustics, the first echo is usually used to estimate the input signal to the system and the rest of the waveform is considered as the measured output. However, when examining multi-layered structures, a first separable echo might not be present. This is usually the case when the top layer is thin and the transducer produces a wave with time support too long relative to the layer thickness, which is a common problem when using low-frequency transducers. In this situation, the measured signal waveform consists of several overlapping and reverberant echoes.

When the first echo is not completely separable from the rest of the signal waveform, there are 2 main approaches. One is to use a buffer rod between the transducer and the material that is thick enough to get a separate echo before the signal waveform from the material is received. The major advantage with this solution is the preservation of the measurement conditions. The drawback with this solution is a loss in energy at the boundary between the buffer rod and the material, and hence less energy is received from the material under investigation. Another drawback when measuring on solid material is the introduction of undesirable reflections, because there might be a gap between the buffer rod and the material. The second solution is to retrieve the input signal from a 
different measurement. The idea is to use a thicker layer of the same material as the top layer in the material under investigation. The drawback with this solution is the difficulty in achieving the same measurement conditions for the 2 experiments, leading to temperature and pressure differences and misalignment effects. Consideration of pre-alignment and appropriate scaling of the input signal must therefore be taken. The effect of this misalignment may be a small spreading of the echoes, resulting in a slightly different waveform.

In a through-transmission configuration, the first echo cannot be used to estimate the input signal because the received echo has already been affected by the material, which is undesirable when estimating the input signal. Also, if any of the layers are thin, the first echo is not separable from the rest of the measured waveform. One suggestion is to use a pulse-echo measurement, following the procedure mentioned above, to estimate the input signal, but with the drawback that different receivers are used when measuring the input and output signals. Another solution is to remove the specimen under investigation to perform the measurement used to estimate the input signal. This solution still involves some difficulties. The differences in phase velocities inside the materials in the separate measurements imply different diffraction affecting the appearance of the echoes. Also, when dealing with a material with a highly frequency-dependent attenuation, the shape of the waveforms will differ. A solution to this is to use a thick sample of the same material, and perform a through-transmission measurement to capture a signal that has undergone approximately the same condition as the measured output signal. The specific measurement conditions in the experiment and the sample under investigation is the basis for the decision on which method to use.

\section{The LAYer ModeL}

Sending an ultrasonic pulse through a multi-layered material will produce a received output signal waveform consisting of several delayed and attenuated echoes. If the multi-layered structure has 1 or more thin layers, the signal waveform will consist of overlapping echoes. Several different solutions to handle multi-layered structures have been proposed [8], [9]. In this study, a system identification approach is used, where the multi-layered structure is described by a parametric model. The ultrasonic signal waveform that has propagated through a multi-layered structure with an arbitrary number of layers is measured. A system of linear equations is used to derive the transfer functions between the defined input and output signals, valid for through-transmission and pulse-echo setups, or both setups simultaneously. The multi-layered model is derived in the frequency domain; for clarity, however, most signal waveforms in the figures are shown in the time domain.

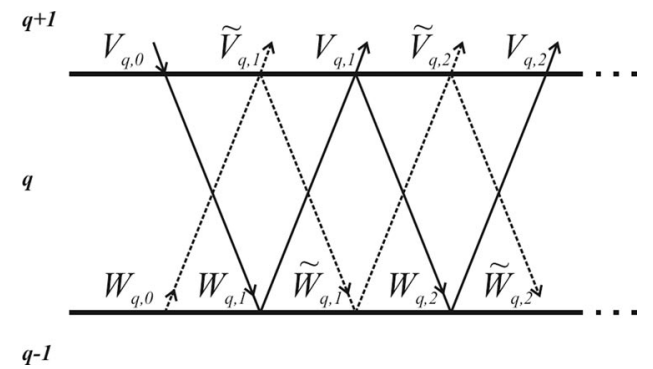

Fig. 3. A single layer in a multi-layer structure: $V_{q, 0}$ represents the input signal from the layer above; $W_{q, 0}$ represents the input signal from the layer below; $V_{q, p}, \tilde{V}_{q, p}, W_{q, p}$, and $\tilde{W}_{q, p}$ for $p=1,2, \ldots, \infty$, (in this figure, $p=1,2$ is visible) represent the output echoes from the layer.

\section{A. Single Layer in a Multi-Layered Structure}

The model for a medium with a multi-layered structure is found by examining the reflections of the input signals from inside the medium. The complexity in finding a model that describes all echoes from the layers increases rapidly as the number of layers increases. Therefore, a method to find the model structure for arbitrary number of layers is desirable. The approach is to find the model for a single layer within a multi-layered structure, and model every individual layer in the multi-layered structure accordingly.

In a single layer inside a multi-layered structure, the input echoes to that layer are arriving from both boundaries, i.e., from the top and from the bottom layer as shown in Fig. 3. In the following derivation of the layer model, $\omega$ is omitted for notational simplicity. The input signal $V_{q, 0}$ will contribute to the output echoes at both boundaries. The contributions at the upper boundary are

$$
\begin{gathered}
V_{q, 1}=T_{q+1, q} R_{q, q-1} T_{q, q+1} M_{q}^{2} V_{q, 0} \\
V_{q, 2}=R_{q, q-1} R_{q, q+1} M_{q}^{2} T_{q+1, q} R_{q, q-1} T_{q, q+1} M_{q}^{2} V_{q, 0} .
\end{gathered}
$$

$$
V_{q, p}=R_{q, q-1} R_{q, q+1} M_{q}^{2} V_{q, p-1}, \quad \text { for } p \geq 2
$$

where $p=1,2, \ldots, \infty$ enumerates the echoes. The $p$ th echo at the upper boundary can be written as [21]

$$
V_{q, p}=\left(R_{q, q-1} R_{q, q+1} M_{q}^{2}\right)^{p-1} T_{q+1, q} R_{q, q-1} T_{q, q+1} M_{q}^{2} V_{q, 0} .
$$

The input signal $W_{q, 0}$ will also contribute to the output echoes at both boundaries. The $p$ th echo at the upper boundary created from this input can be written as

$$
\tilde{V}_{q, p}=\left(R_{q, q-1} R_{q, q+1} M_{q}^{2}\right)^{p-1} T_{q, q+1} M_{q} W_{q, 0} .
$$

The input signals will also produce output echoes at the lower boundary. The $p$ th echo at the lower boundary can be written as 


$$
\begin{gathered}
W_{q, p}=\left(R_{q, q-1} R_{q, q+1} M_{q}^{2}\right)^{p-1} T_{q+1, q} M_{q} V_{q, 0}, \\
\tilde{W}_{q, p}=\left(R_{q, q-1} R_{q, q+1} M_{q}^{2}\right)^{p-1} R_{q+1, q} M_{q}^{2} W_{q, 0} .
\end{gathered}
$$

The 0 in the input signals $V_{q, 0}$ and $W_{q, 0}$, denotes that the total output from layer $q$ at the upper boundary is defined as the first echo in layer $(q+1)$, denoted $W_{q+1,0}$.

The upward output signal is the sum of all pressure waves leaving the $q$ th layer into the $(q+1)$ th layer. This means that this output is the lower input signal $W_{q+1,0}$ to the $(q+1)$ th layer. Using the expressions in (12) and (13), the upward output can be expressed as

$$
\begin{aligned}
& W_{q+1,0}=\sum_{p=1}^{\infty}\left(V_{q, p}+\tilde{V}_{q, p}\right) \\
& \quad=\frac{T_{q+1, q} R_{q, q-1} T_{q, q+1} M_{q}^{2}}{1-R_{q, q-1} R_{q, q+1} M_{q}^{2}} V_{q, 0}+\frac{T_{q, q+1} M_{q}}{1-R_{q, q-1} R_{q, q+1} M_{q}^{2}} W_{q, 0} \\
& =A_{q} V_{q, 0}+B_{q} W_{q, 0},
\end{aligned}
$$

where the second equalities are given by the geometrical progression

$$
\sum_{n=0}^{\infty} x^{n}=\frac{1}{1-x}
$$

where the condition $|x|<1$ must be fulfilled to guarantee stability in the model.

The downward output signal is the sum of all pressure waves leaving the $q$ th layer into the $(q-1)$ th layer. This means that this output is the upper input signal $V_{q-1,0}$ to the $(q-1)$ th layer. Using the expressions in (14) and (15), the downward output can be expressed as

$$
\begin{aligned}
& V_{q-1,0}=\sum_{p=1}^{\infty}\left(W_{q, p}+\tilde{W}_{q, p}\right) \\
& \quad=\frac{T_{q+1, q} M_{q}}{1-R_{q, q-1} R_{q, q+1} M_{q}^{2}} V_{q, 0}+\frac{R_{q, q+1} M_{q}^{2}}{1-R_{q, q-1} R_{q, q+1} M_{q}^{2}} W_{q, 0} \\
& \quad=C_{q} V_{q, 0}+D_{q} W_{q, 0} .
\end{aligned}
$$

The expressions in (16) and (17) can be rewritten to facilitate the parametrization using the relationships $T_{l, k}=$ $1+R_{l, k}$ and $R_{k, l}=-R_{l, k}$

$$
\begin{gathered}
A_{q}=\frac{\left(1-R_{q+1, q}^{2}\right) R_{q, q-1} M_{q}^{2}}{1+R_{q, q-1} R_{q+1, q} M_{q}^{2}}, \\
B_{q}=\frac{\left(1-R_{q+1, q}\right) M_{q}}{1+R_{q, q-1} R_{q+1, q} M_{q}^{2}}, \\
C_{q}=\frac{\left(1+R_{q+1, q}\right) M_{q}}{1+R_{q, q-1} R_{q+1, q} M_{q}^{2}},
\end{gathered}
$$

$$
D_{q}=\frac{-R_{q+1, q} M_{q}^{2}}{1+R_{q, q-1} R_{q+1, q} M_{q}^{2}}
$$

By using (16)-(22), the appropriate model for a multilayered structure with an arbitrary (but known) number of layers, $Q$, can be found. The model can be derived with input- and output signals above and/or under the material; that is, pulse-echo or through-transmission configuration in both directions or both jointly.

\section{B. One-Layered Structure}

The model for a medium with 1 layer, $Q=1$, is found by using the equations derived in the previous section. In a pulse-echo configuration with input and output signal above the material, $V_{1,0}$ is the input signal and $W_{1,0}$ is zero. The output signal $V_{0,0}$ is not interesting and $W_{2,0}$ is the interesting output signal, composed by the reflected echoes, $V_{1, p}$, see Fig. 4 .

The model structure is found from (16) for $Q=1$, using the knowledge that the input from the layers below are zero, $W_{1,0}=0$,

$$
\begin{aligned}
W_{2,0} & =A_{1} V_{1,0}+B_{1} W_{1,0} \\
& =A_{1} V_{1,0}
\end{aligned}
$$

This equation gives us the model for a pulse-echo configuration with input and output signal above the material

$$
\begin{gathered}
\mathrm{H}_{1}(\omega)=A_{1}(\omega) \\
=\frac{\left(1-R_{21}^{2}\right) R_{10} M_{1}^{2}(\omega)}{1+R_{10} R_{21} M_{1}^{2}(\omega)},
\end{gathered}
$$

where $M_{1}(\omega)$ is the collected material properties in the single layer defined in (6). This is the model for a 1-layer structure in pulse-echo configuration with transmitter and receiver above the material. Using (16) and (18) in ways similar to those described previously, the models for both pulse-echo and through-transmission configuration can be found. For a 1-layer structure, the total model can be stated using a system of linear equations in a block matrix as

$$
\left[\begin{array}{l}
W_{2,0} \\
V_{0,0}
\end{array}\right]=\left[\begin{array}{ll}
A_{1} & B_{1} \\
C_{1} & D_{1}
\end{array}\right]\left[\begin{array}{c}
V_{1,0} \\
W_{1,0}
\end{array}\right],
$$

where the different choices of input and output signals give the desired model. Observe that when removing the second row and column in the matrix, the model for the pulse-echo configuration in (23) is achieved. A similar matrix of linear equations can be built up, extending the model for 2,3 , and more layers. 


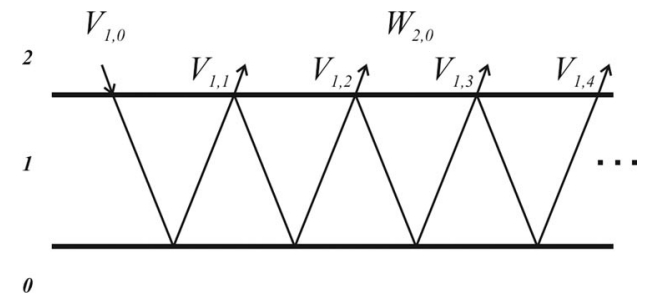

Fig. 4. A 1-layer structure in a pulse-echo configuration: $V_{1,0}$ represents the input signal from the layer above; $W_{2,0}$ represents the total output signal from the layer; $V_{1, p}$ for $p=1,2, \ldots, \infty$, represents the output echoes from the layer.

\section{Two-Layered Structure}

The model for a medium with 2 layers, $Q=2$, is found using the same approach as for a medium with 1 layer, by examining the reflections of the input signal from inside the medium. This is done by examining the equations in Section III-A. However, equivalent to the system of linear equations in the matrix in (26), a matrix can be found for the 2-layered structure by expanding for another layer

$$
\left[\begin{array}{c}
W_{3,0} \\
V_{1,0} \\
W_{2,0} \\
V_{0,0}
\end{array}\right]=\left[\begin{array}{cccc}
A_{2} & B_{2} & 0 & 0 \\
C_{2} & D_{2} & 0 & 0 \\
0 & 0 & A_{1} & B_{1} \\
0 & 0 & C_{1} & D_{1}
\end{array}\right]\left[\begin{array}{c}
V_{2,0} \\
W_{2,0} \\
V_{1,0} \\
W_{1,0}
\end{array}\right] .
$$

In a pulse-echo configuration with input and output signals above the material, $V_{2,0}$ is the input signal and $W_{1,0}$ is zero. The output signal $V_{0,0}$ is not interesting, and $W_{3,0}$ is the interesting output signal, see Fig. 5. For clarity, all reflected echoes are not shown in this figure. The dashed lines (waves) at the top surface are reflected back down in the structure and will give rise to additional reflected echoes. All these reverberation are included in the model and rapidly complicate it.

By using the knowledge of a pulse-echo configuration, the system of linear equations in (27) reduces to

$$
\left[\begin{array}{c}
W_{3,0} \\
V_{1,0} \\
W_{2,0}
\end{array}\right]=\left[\begin{array}{ccc}
A_{2} & B_{2} & 0 \\
C_{2} & D_{2} & 0 \\
0 & 0 & A_{1}
\end{array}\right]\left[\begin{array}{c}
V_{2,0} \\
W_{2,0} \\
V_{1,0}
\end{array}\right],
$$

with $W_{3,0}$ as output signal and $V_{2,0}$ as input signal. The signals $V_{1,0}$ and $W_{2,0}$ are the input and output at the middle interface between the first and second layer, respectively. These signals are used in the calculations and guarantee that all reverberations are taken into account as they include the terms $A_{1}, B_{2}, C_{2}$, and $D_{2}$ in the model, which contain the internal reverberations, see (19)-(22). Solving this system of linear equations with respect to the output and input signals will give us the model for a 2-layered structure. The linear equations are,

$$
\begin{aligned}
& W_{3,0}=A_{2} V_{2,0}+B_{2} W_{2,0}, \\
& V_{1,0}=C_{2} V_{2,0}+D_{2} W_{2,0}
\end{aligned}
$$

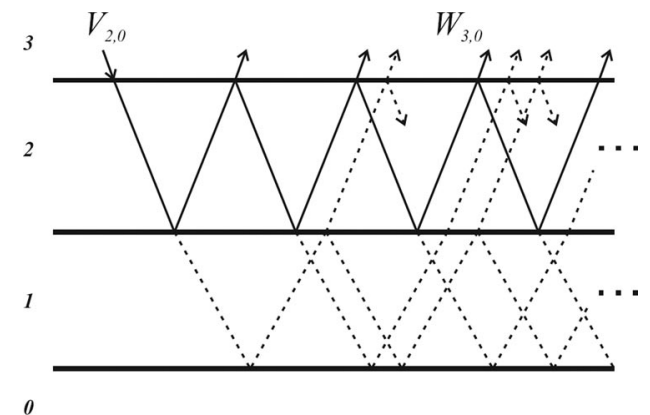

Fig. 5. A 2-layer structure: $V_{2,0}$ represents the input signal from the layer above; $W_{3,0}$ represents the output signal from the material consisting of 2 layers.

$$
W_{2,0}=A_{1} V_{1,0}
$$

To find the output signal $W_{3,0}$ expressed in the input signal $V_{2,0}$, insert (30) into (31) and solve for $W_{2,0}$; insert the result into (29), and the result is

$$
W_{3,0}=A_{2} V_{2,0}+B_{2} \frac{C_{2} A_{1}}{1-D_{2} A_{1}} V_{2,0} .
$$

This equation gives us the model for a pulse-echo configuration with both input and output signals above the material

$$
\mathrm{H}_{2}(\omega)=A_{2}(\omega)+\frac{B_{2}(\omega) C_{2}(\omega) A_{1}(\omega)}{1-D_{2}(\omega) A_{1}(\omega)} .
$$

\section{Summary of the Multi-Layered Model}

To find the transfer function between the inputs $V_{Q}$ and $W_{1}$ and the outputs $W_{Q+1}$ and $V_{0}$, a system of linear equations must be solved, obtained from the equations in Section III-A. For $Q$ layers, see Fig. 6, the linear system can be expressed as

$$
\left[\begin{array}{c}
W_{Q+1} \\
V_{Q-1} \\
\vdots \\
W_{3} \\
V_{1} \\
W_{2} \\
V_{0}
\end{array}\right]=\left[\begin{array}{ccccccc}
A_{Q} & B_{Q} & 0 & & & \cdots & 0 \\
C_{Q} & D_{Q} & 0 & & & & \vdots \\
0 & 0 & \ddots & 0 & 0 & & \\
& & 0 & A_{2} & B_{2} & 0 & 0 \\
& & 0 & C_{2} & D_{2} & 0 & 0 \\
\vdots & & & 0 & 0 & A_{1} & B_{1} \\
0 & \cdots & & 0 & 0 & C_{1} & D_{1}
\end{array}\right]\left[\begin{array}{c}
V_{Q} \\
W_{Q} \\
\vdots \\
V_{2} \\
W_{2} \\
V_{1} \\
W_{1}
\end{array}\right] .
$$

The task is to find the complete transfer function $\tilde{\mathbf{H}}$ for the layered structure

$$
\tilde{\mathbf{y}}=\tilde{\mathbf{H}} \tilde{\mathbf{u}},
$$

where

$$
\tilde{\mathbf{y}}=\left[\begin{array}{c}
W_{Q+1} \\
V_{0}
\end{array}\right], \quad \tilde{\mathbf{H}}=\left[\begin{array}{cc}
\tilde{h}_{1,1} & \tilde{h}_{1,2} \\
\tilde{h}_{2,1} & \tilde{h}_{2,2}
\end{array}\right], \quad \tilde{\mathbf{u}}=\left[\begin{array}{c}
V_{Q} \\
W_{1}
\end{array}\right]
$$




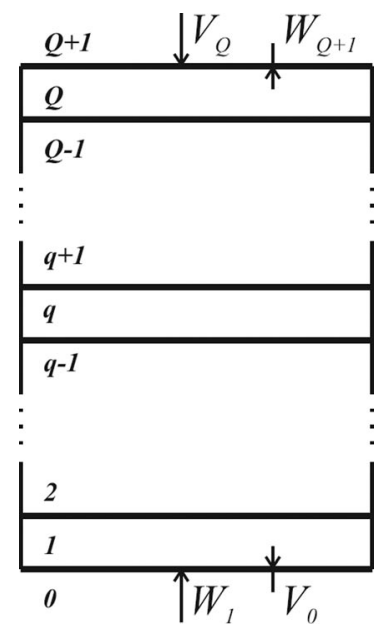

Fig. 6. A multi-layered structure with $Q$ layers, with the inputs and outputs at the boundaries.

where $V_{Q}$ is defined as the input at the upper boundary, $W_{1}$ is defined as the input at the lower boundary, $W_{Q+1}$ is the output at the upper boundary, and $V_{0}$ is the output at the lower boundary. The transfer functions $\tilde{h}_{k, l}$ can be derived from the matrix relationship in (34).

\section{E. Recursive Solution to $\tilde{h}_{1,1}$ in $\tilde{\mathbf{H}}$}

The expression $\tilde{h}_{1,1}$ in (36) is in question when only the input and output signal are at the top of the layers, and the input and output signal at the bottom of the layers are missing (pulse-echo configuration with transmitter and receiver above the structure). This specific measurement condition has been evaluated in previous studies, see [22] [24], and it has been shown that it is possible to find a recursive expression for the model, when using this measurement condition. The expression $\tilde{h}_{1,1}$ for $Q$ layers can be found using the recursive expression

$$
\mathrm{H}_{q}= \begin{cases}A_{q}+\frac{B_{q} C_{q} \mathrm{H}_{q-1}}{1-D_{q} \mathrm{H}_{q-1}}, & q>1 \\ A_{1}, & q=1 .\end{cases}
$$

This expression follows from the linear equations in (34) and facilitates the derivation of the multi-layered model for an arbitrary number of layers. The models for 1-, and 2-layered structures in pulse-echo configuration given in (24) and (33) follow from this equation.

\section{F. Parametrization of the Model}

The model is parameterized by several unknown parameters collected in the parameter vector $\boldsymbol{\theta}$. The general model for $Q$ layers is expressed as $\mathrm{H}_{Q}(\omega, \boldsymbol{\theta})$, where $\boldsymbol{\theta}$ contains the reflection coefficients at the boundaries between the layers, the time of flight and the attenuation coefficient from $M_{q}(\omega)$ for each layer. For $Q$ layers the parameter vector is $\boldsymbol{\theta}=\left[R_{Q+1, Q}, \ldots, R_{21}, R_{10}, \tau_{Q}, \ldots, \tau_{2}, \tau_{1}, \alpha_{Q}, \ldots, \alpha_{2}\right.$, $\left.\alpha_{1}\right]^{T}$, where all parameters are connected to acoustic properties in the material, see Sections II-B and II-C.

\section{G. Model Validation}

To make sure that the parametric model is capable of describing the multi-layered structure, we have to evaluate its performance. The key concept here is to examine the residuals (the part of the response $y(t)$ that the model could not reproduce) to determine the model's validity [25]. If the residuals do not contain components other than measurement noise, the conclusion is that the model can be used to describe the multi-layered structure.

\section{Maximum Likelihood Estimation of the Model Parameters}

In this section, the parameter estimation and the optimization algorithm are given. Finally, a stepwise explanation of the algorithm is presented.

\section{A. Parameter Estimation and Optimization}

The model parameters are estimated by deriving the maximum likelihood estimator (MLE) for the parameters [26]. The noise on both the input signals and the output signals is used to weight the model error, implying that the MLE ends up in a nonlinear weighted least squares (NWLS) problem. The unknown model parameters are estimated from discrete Fourier-transformed data [20]. The objective of the parameter estimation is to find the parameters that minimize the square of the error, weighted by the covariance matrix. Because of the nonlinearity with respect to the parameter vector $\boldsymbol{\theta}$, finding the minimum must be based on numerical iterative approaches. The weighted error $\varepsilon\left(\omega_{k}, \boldsymbol{\theta}\right)$ of the model that is to be minimized is

$$
\varepsilon\left(\omega_{k}, \boldsymbol{\theta}\right)=\frac{\mathrm{Y}\left(\omega_{k}\right)-\mathrm{H}\left(\omega_{k}, \boldsymbol{\theta}\right) \mathrm{U}\left(\omega_{k}\right)}{\sigma_{e}\left(\omega_{k}, \boldsymbol{\theta}\right)},
$$

for the $k$ frequency points, where $\sigma_{e}^{2}(\omega, \boldsymbol{\theta})$ is the variance of the error [20], see Appendix A-C. Note that if $\varepsilon(\omega, \boldsymbol{\theta}) \sim \mathcal{N}\left(\mathbf{0}, \sigma^{2} \mathbf{I}\right)$, the NWLS is also the MLE. The NWLS estimator is found by minimizing the LS error function

$$
J(\boldsymbol{\theta})=\varepsilon(\boldsymbol{\theta})^{H} \varepsilon(\boldsymbol{\theta}),
$$

where $\varepsilon(\boldsymbol{\theta})$ is a vector containing the errors for all frequencies, defined in (38), and ${ }^{H}$ is the Hermitian transpose. The iterative update of the parameter vector to find the minimum of (39) is performed by using the Gauss-Newton linearization method [26], see Appendix A-C for details. The Gauss-Newton method requires the Jacobian (gradients) and an approximation of the Hessian of $J(\boldsymbol{\theta})$ in (39), see Appendix A-B. The Gauss-Newton linearization algorithm that is used to iteratively find the parameter vector $\boldsymbol{\theta}^{\{i+1\}}$, by updating $\boldsymbol{\theta}^{\{i\}}$ with $\Delta \boldsymbol{\theta}^{\{i\}}$, is then given by [26] 


$$
\boldsymbol{\theta}^{\{i+1\}}=\boldsymbol{\theta}^{\{i\}}-\Delta \boldsymbol{\theta}^{\{i\}},
$$

where

$$
\Delta \boldsymbol{\theta}=\left(\Re\left\{\frac{\partial \varepsilon^{H}}{\partial \boldsymbol{\theta}} \frac{\partial \varepsilon}{\partial \boldsymbol{\theta}}\right\}\right)^{-1} \cdot \Re\left\{\frac{\partial \varepsilon^{H}}{\partial \boldsymbol{\theta}} \varepsilon\right\}
$$

using $\boldsymbol{\theta}=\boldsymbol{\theta}^{\{i\}}$ and where the gradient $\partial \varepsilon / \partial \boldsymbol{\theta}$ is found in Appendix A-C. To speed up the parameter estimation algorithm, we need the gradient of $\mathrm{H}(\omega, \boldsymbol{\theta})$, and calculating the gradient is essential for the algorithm. The gradient of $\mathrm{H}(\omega, \boldsymbol{\theta})$ is calculated with respect to every parameter in the parameter vector $\boldsymbol{\theta}$ [24].

In the estimation process, an initial guess of the values of the parameter vector is required. A poor initial guess could result in a local minimum and consequently an inaccurate parameter estimation. Because the parameters are connected to physical properties, an initial guess can sometimes be taken from theoretical values but also from information taken from the measured signals.

In addition to the Gauss-Newton algorithm, the Hessian is also regularized, using the Levenberg-Marquardt optimization [27], [28], to get a more stable parameter estimation algorithm and to expand the convergence region [29]. Adding the regularization to the update of the parameter vector in (41) gives

$$
\Delta \boldsymbol{\theta}=\left(\Re\left\{\frac{\partial \varepsilon^{H}}{\partial \boldsymbol{\theta}} \frac{\partial \varepsilon}{\partial \boldsymbol{\theta}}\right\}+\lambda^{2} \mathbf{I}\right)^{-1} \cdot \Re\left\{\frac{\partial \varepsilon^{H}}{\partial \theta} \varepsilon\right\},
$$

using $\boldsymbol{\theta}=\boldsymbol{\theta}^{\{i\}}$, and where $\mathbf{I}$ is the identity matrix and $\lambda$ is a scalar controlling the regularization. When $\lambda$ is zero, the algorithm uses the ordinary Gauss-Newton method; and when $\lambda$ goes to infinity, the steepest gradient descent minimization is used. The scalar $\lambda$ is updated in every iteration as [20]

$$
\lambda_{i+1}= \begin{cases}0.4 \lambda_{i}, & \text { if } J\left(\boldsymbol{\theta}^{\{i\}}\right)<J\left(\boldsymbol{\theta}^{\{i-1\}}\right) \\ 10 \lambda_{i}, & \text { if } J\left(\boldsymbol{\theta}^{\{i\}}\right) \geq J\left(\boldsymbol{\theta}^{\{i-1\}}\right)\end{cases}
$$

and $\lambda_{0}$ is equal to the largest singular value of the cost function $J\left(\boldsymbol{\theta}^{\{0\}}\right)$ in (39).

\section{B. Parameter Estimation Algorithm}

When implementing the Gauss-Newton algorithm, the gradient is calculated analytically to speed up computations. The iteration is terminated when either the norm of the improvement or the descent of the cost function are smaller than predefined thresholds. The Gauss-Newton parameter estimation algorithm can be implemented in the following steps:

- Step 1: Guess the initial values of the parameters in the parameter vector $\boldsymbol{\theta}^{\{0\}}$ and set the iteration number $i=0$.
- Step 2: Compute the transfer function $\mathrm{H}\left(\omega, \boldsymbol{\theta}^{\{i\}}\right)$ using the layer model in (34) and the specific measurement conditions. Compute the error $\varepsilon\left(\omega, \boldsymbol{\theta}^{\{i\}}\right)$ in (38), and the gradient $\partial \varepsilon\left(\omega, \boldsymbol{\theta}^{\{i\}}\right) / \partial \boldsymbol{\theta}^{\{i\}}$ in (55).

- Step 3: Iterate the parameter vector $\boldsymbol{\theta}^{\{i+1\}}$ in (41) using the Levenberg-Marquardt optimization in (42).

- Step 4: Check convergence criterions and break if either $\left\|\boldsymbol{\theta}^{\{i+1\}}-\boldsymbol{\theta}^{\{i\}}\right\|<\operatorname{threshold}_{1}$ or $J\left(\boldsymbol{\theta}^{\{i-1\}}\right)-J\left(\boldsymbol{\theta}^{\{i\}}\right)$ $<$ threshold $_{2}$.

- Step 5: If not converged, set $i \rightarrow i+1$ and go to Step 2.

\section{Experimental Setup}

All measurements were conducted with the ultrasound transducer immersed in water and with the specimen surrounded by water. The measurements were performed in a combined pulse-echo and through-transmission configuration, see Fig. 7. The temperature in the water was kept constant at $20.15^{\circ} \mathrm{C}$ to within $\pm 0.05^{\circ} \mathrm{C}$.

The examined specimen consisted of 2 Pyrex glass layers bonded together by a thin layer. The thickness of the top glass layer was $1.83 \pm 0.05 \mathrm{~mm}$ and the thickness of the bottom glass layer was $3.78 \pm 0.05 \mathrm{~mm}$. Tabulated values of speed of sound and density in the Pyrex glass layers are $c=5600 \mathrm{~m} / \mathrm{s}$ and $\rho=2300 \mathrm{~kg} / \mathrm{m}^{3}$ [18], which also can be used to find elastic properties. The thickness of the bonding layer was $0.53 \pm 0.05 \mathrm{~mm}$ and 2 different materials were used for bonding: construction silicone and epoxy. The exact mixtures and degree of curing is unknown for these materials in this study.

For all the experiments performed in this work, a $5-\mathrm{MHz}$ unfocused piezoelectric transducer (V3456, Panametrics, Waltham, MA) with an element size of $13 \mathrm{~mm}$ was used. With this applied ultrasonic frequency, the thickness of the thin embedded middle layer in the experimental study is in the order of the wavelength of the ultrasonic wave in that layer. To excite and receive acoustic pulses, a Panametrics Pulser/Receiver Model 5073PR, operating in both pulse-echo and through-transmission mode was used. The acquired ultrasonic echoes were sampled using a Gage CompuScope CS12400 (Lachine, QC, Canada), at $200 \mathrm{MHz}$ with a 12-bit resolution.

In the through-transmission measurements, a needle-type miniature PVDF ultrasonic hydrophone probe (MHA9-150, FORCE Technology, Västerås, Sweden), was used as the receiver. The active element on the hydrophone is a $9 \mu \mathrm{m}$ PVDF film with gold electrodes and the hydrophone tip has an effective radius of $150 \mu \mathrm{m}$ and a frequency range from $100 \mathrm{kHz}$ up to $80 \mathrm{MHz}$.

\section{Measurement Results}

\section{A. Pulse-Echo Configuration}

In this experiment, we use a 3-layered material with a relatively thin middle layer of epoxy. A pulse-echo con- 


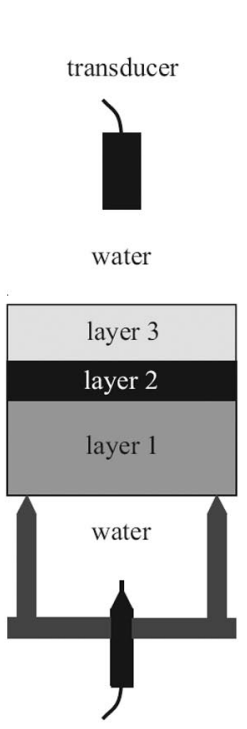

hydrophone

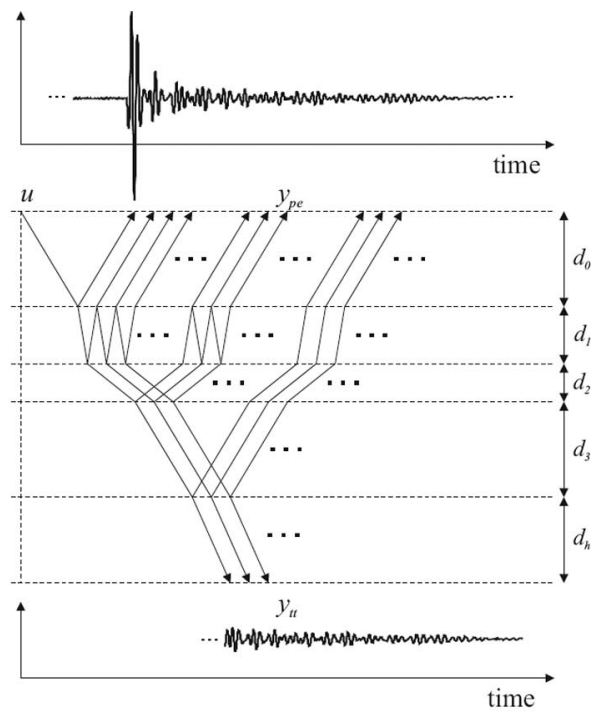

me

Fig. 7. The experimental setup in a pulse-echo and through-transmission configuration. The waveforms and the notations are in the time domain, where $u(t)$ represents the input signal, $y_{\mathrm{pe}}(t)$ is the entire output signal in pulse-echo measurements, and $y_{\mathrm{tt}}(t)$ is the entire output signal in through-transmission measurements.

figuration is used, and the measured waveform consists of overlapping reverberant echoes. In Fig. 8(a), the measured output signal from a thin 3-layered material is shown. The waveform consists of multiple overlapping echoes, and the first echo is not separable from the others. A reference measurement on a material with a thicker top layer is conducted, implying a separable first echo, see Fig. 8(b). This measurement is essential to capture an input signal. The input signal is captured by windowing the first echo and performing an appropriate scaling and a pre-alignment before the estimation of the input at the transducer is performed. The prepared first echo is then used to create the input signal as

$$
\mathrm{U}(\omega)=\frac{1}{R_{01}} e^{j \omega 2 \tau_{0}} \mathrm{U}_{1}(\omega),
$$

where $\mathrm{U}_{1}(\omega)$, shown in Fig. 8(c), is taken from the first echo in the separate measurement and $\tau_{0}$ is the time-offlight in the water between the transducer and the material.

In pulse-echo configuration, the linear system in (7) uses the input signal, $\mathrm{U}(\omega)$, given by (44), and the transfer function given by

$$
\mathrm{H}(\omega)=\left(R_{43}+\mathrm{H}_{3}(\omega)\right) e^{-j \omega 2 \tau_{0}},
$$

where $\mathrm{H}_{3}(\omega)$ is calculated from $\tilde{h}_{1,1}$ in (36). In Fig. 2(a), the measured output signal is visible as the dashed line. Using the model and the estimated parameters together with the estimated input signal, we are then capable of reconstructing the measured output signal. The estimated output signal is shown in Fig. 2(a) as a solid line. The fit

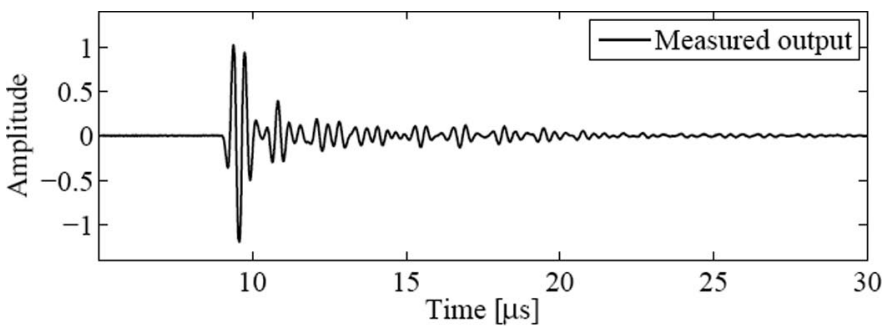

(a)

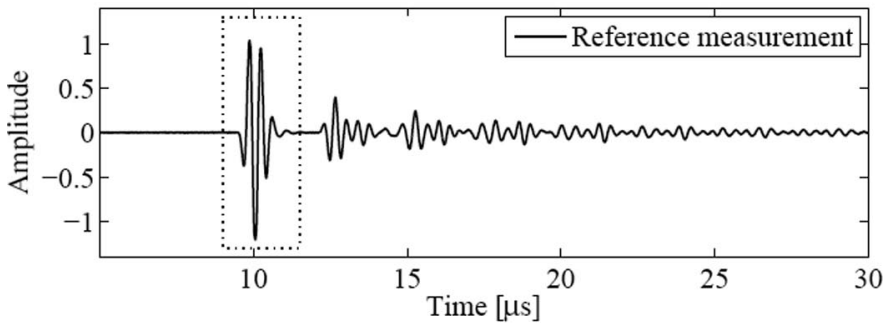

(b)

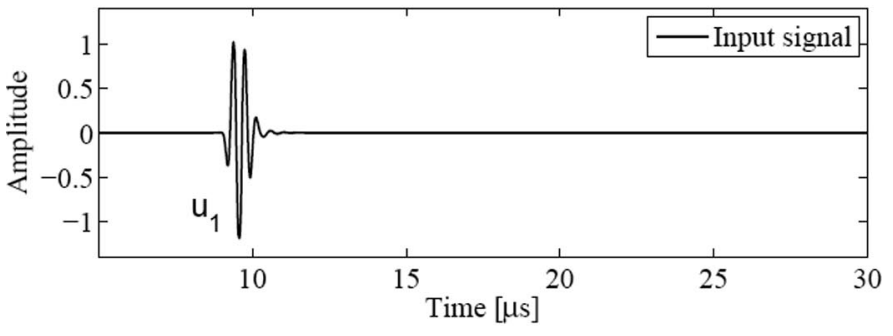

(c)

Fig. 8. In (a) the measured output signal from a thin 3-layered material, with epoxy as the bonding material, is shown. The waveform consists of multiple overlapping echoes and the first echo is not separable from the others. The waveform in (b) is from a sample with a thicker top layer, implying a separable first echo. In (c) the windowed, scaled and prealigned echo that is used to estimate the input signal is shown.

between the measured and the estimated signal is very good. To further analyze the estimation result, the residual is examined, see Fig. 2(b). Investigating the $y$-axis reveals that the scale is decreased and that the residual is small. Some systematic variations are still left, indicating that this hard physical model does not capture all dynamics. However, the residual contains only small variations.

The estimated waveform in Fig. 9(a) is recreated using the estimated parameters, see Table I.

\section{B. Through-Transmission Configuration}

In this experiment we use the same 3-layered material with a relatively thin middle layer of epoxy. In this setup, a through-transmission configuration is used, and the measured waveform is received by a hydrophone under the material. Again, the received waveform consists of overlapping reverberant echoes. In Fig. 10(a), the measured output signal from the thin 3-layered material is shown. The waveform consists of multiple overlapping echoes, and the first echo is not separable from the others. This waveform, compared with the waveform from a pulse-echo configuration, consists of even more overlapping echoes. 


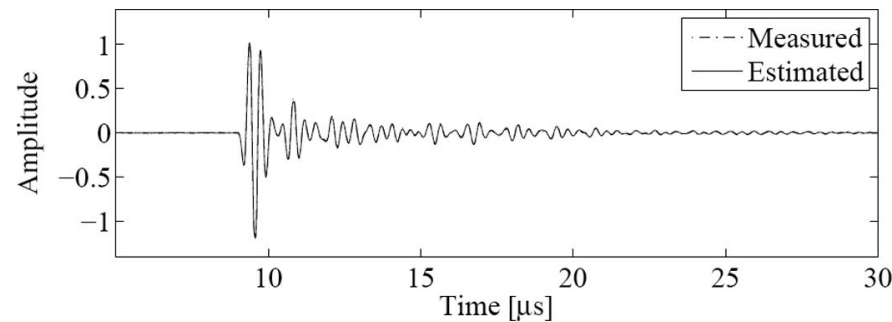

(a)

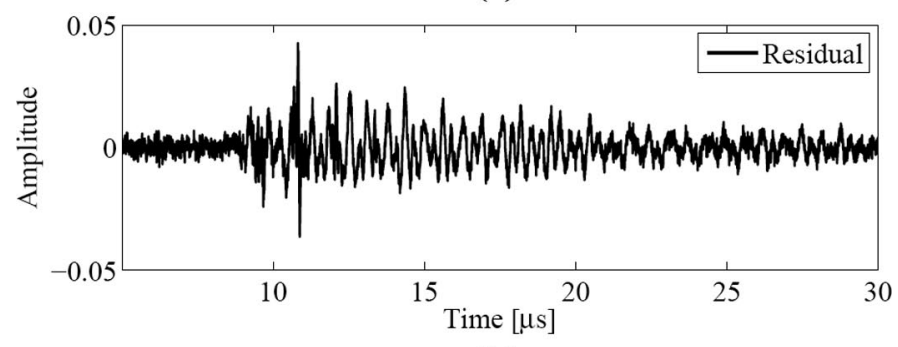

(b)

Fig. 9. (a) The measured output signal, $y_{\mathrm{m}}(t)$, from a thin 3-layered material and the estimated output signal, $y_{\mathrm{e}}(t)$, (b) the residual $e(t)=$ $y_{\mathrm{m}}(t)-y_{\mathrm{e}}(t)$

TABle I. The Estimated Parameters $\theta$ For the Pulse-Echo Experiment With Epoxy as the Bonding Material.

\begin{tabular}{llc}
\hline$\hat{R}_{43}=0.8056$ & $\hat{\tau}_{3}=126.48$ & $\hat{\alpha}_{3}=445.79$ \\
$\hat{R}_{32}=-0.7136$ & $\hat{\tau}_{2}=90.729$ & $\hat{\alpha}_{2}=54706$ \\
$\hat{R}_{21}=0.7138$ & $\hat{\tau}_{1}=260.62$ & $\hat{\alpha}_{1}=419.15$ \\
$\hat{R}_{10}=-0.8058$ & & \\
\hline
\end{tabular}

This is because all echoes have traveled through all layers at least once, compared with the pulse-echo measurements for which some echoes just travel in the top layer(s). However, a reference measurement without a material between the transmitter and the receiver is conducted, implying a completely separated echo, see Fig. 10(b). This measurement is essential to capture an input signal. The input signal is captured by performing an appropriate scaling and a pre-alignment before the estimation of the input at the transducer is performed.

The signal $\mathrm{U}_{2}(\omega)$, shown in Fig. $10(\mathrm{c})$, is used to create the input signal as

$$
\mathrm{U}(\omega)=e^{j \omega\left(\tau_{0}+\tau_{m}+\tau_{h}\right)} \mathrm{U}_{2}(\omega),
$$

where $\tau_{0}$ is the time-of-flight in the water between the transducer and the material, $\tau_{h}$ is the time-of flight in the water between the material and the hydrophone, and $\tau_{m}$ is the time-of-flight in the water in the void of the material.

In through-transmission configuration, the linear system in (7) uses the input signal, $\mathrm{U}(\omega)$, given by (46) and the transfer function given by

$$
\mathrm{H}(\omega)=\mathrm{H}_{3}(\omega)\left(1+R_{10}\right) e^{-j \omega\left(\tau_{0}+\tau_{h}\right)},
$$

where $\mathrm{H}_{3}(\omega)$ is calculated from $\tilde{h}_{2,1}$ in (36). In Fig. 11(a), the measured output signal is visible as the dashed line. Using the model and the estimated parameters together
TABLE II. The Estimated Parameters $\theta$ For the ThroughTransmission Experiment With Epoxy as the Bonding MATERIAL.

\begin{tabular}{lcc}
\hline$\hat{R}_{43}=0.7981$ & $\hat{\tau}_{3}=126.4919$ & $\hat{\alpha}_{3}=459.98$ \\
$\hat{R}_{32}=-0.6602$ & $\hat{\tau}_{2}=90.7115$ & $\hat{\alpha}_{2}=54853$ \\
$\hat{R}_{21}=0.6978$ & $\hat{\tau}_{1}=260.6342$ & $\hat{\alpha}_{1}=415.13$ \\
$\hat{R}_{10}=-0.8234$ & & \\
\hline
\end{tabular}

with the estimated input signal, we are then capable of reconstructing the measured output signal. The estimated output signal is shown in Fig. 11(a) as a solid line. The fit between the measured and the estimated signal is quite good. The reconstruction is not as good as in the pulseecho measurement and this is due to the inaccurate input signal estimation. This is visible in Fig. 10, where the appearance of the input echo in Fig. 10(c) differs from the first echo in the measured output waveform in Fig. 10(a). To further analyze the estimation the residual is examined, see Fig. 11(b). Some systematic variations are still left, indicating that this hard physical model does not capture all dynamics. However, the main leftovers are from the deviation in the input signal.

The estimated waveform in Fig. 11(a) is recreated using the estimated parameters, see Table II.

\section{Connecting Model Parameters to Physical Properties}

The estimated parameter vector $\hat{\boldsymbol{\theta}}$ in Tables I and II can be used to directly extract several properties, such as speed of sound, acoustic impedance, and density. Assuming that the thicknesses of the layers are known, the speed of sound for the actual frequency can be estimated as $\hat{c}_{q}=2 d_{q} / \hat{\tau}_{q}$ and the acoustic impedance at the boundary of the layer can be estimated as,

$$
\hat{z}_{q}=\hat{z}_{q+1}\left(\frac{1+\hat{R}_{q+1, q}}{1-\hat{R}_{q+1, q}}\right),
$$

where $\hat{z}_{q+1}$ is the estimated impedance in the layer above. Finally, for $q=Q+1$, the acoustic impedance in the water buffer region is defined as $z_{Q+1}=\rho_{Q+1} c_{Q+1}$, where both the density in water, $\rho_{Q+1}$, and the speed of sound in water, $c_{Q+1}$, are assumed to be known, see [18]. The density can then be estimated as $\hat{\rho}_{q}=\hat{z}_{q} / \hat{c}_{q}$.

In Table III, the estimated speed of sound and the estimated density are presented for the 2 different bonding materials and also for the first glass layer for both measurement configurations. The properties are estimated from 10 repeated measurements, and the mean value and the error in the table are calculated from these experimental measurements. This implies that the repeatability in the estimation is determined by the error. The exact mixture of silicone and epoxy are unknown and hence it is difficult to validate the results. However, the properties for the glass layer are in agreement with previous work, see e.g., [18], [30]. There are some differences between the measurement configurations, and this is because the hy- 


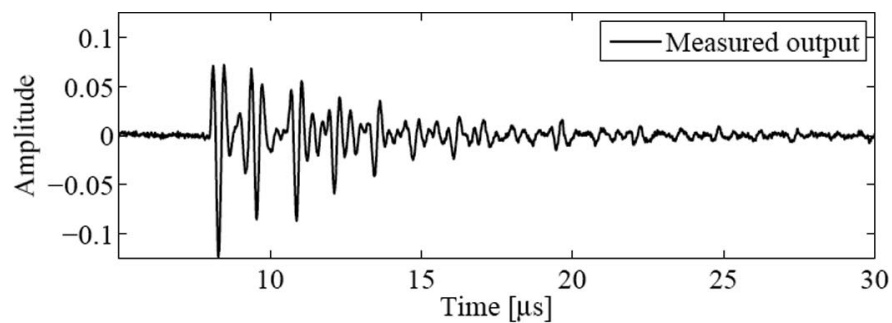

(a)

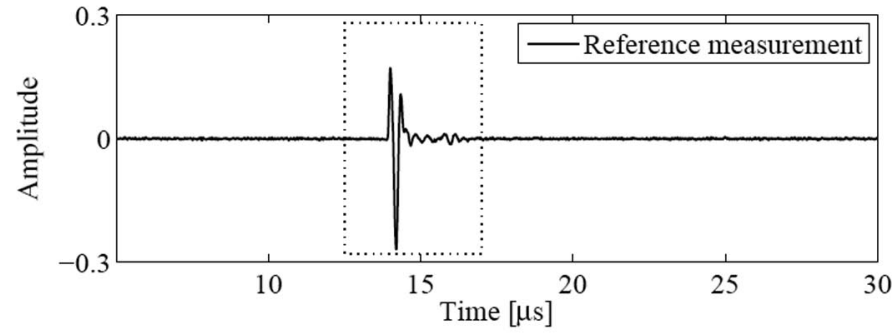

(b)

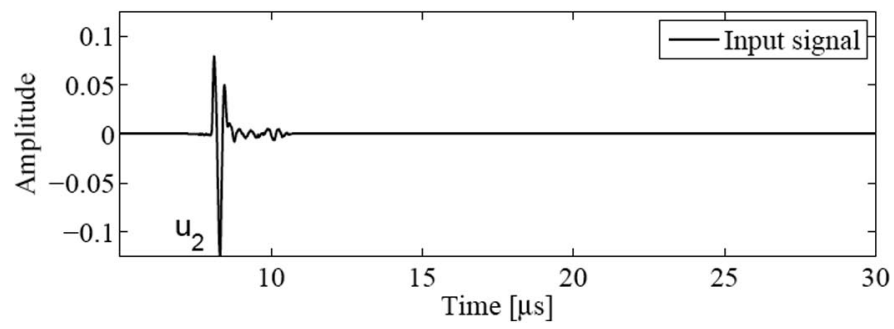

(c)

Fig. 10. (a) The measured output signal from a thin 3-layered material, with epoxy as the bonding material, is shown. The waveform consists of multiple overlapping echoes and the first echo is not separable from the others. The waveform in (b) is from a measurement without a material, implying a separable first echo. In (c), the windowed, scaled and prealigned echo that is used to estimate the input signal is shown.

drophone used as the receiver in the through-transmission configuration has a better spatial resolution then the transducer. This will imply that the through-transmission configuration is more sensitive to local defects. Principally, the speed of sound estimates, $\hat{c}$, are more robust than the density estimates, $\hat{\rho}$. This is because the time-of-flight estimates, $\hat{\tau}_{q}$, in Table I and Table II are very robust.

\section{Discussion}

\section{A. Hard Versus Soft Models}

Sound waves traveling in different media will be affected by the properties of the material, and in a measure-

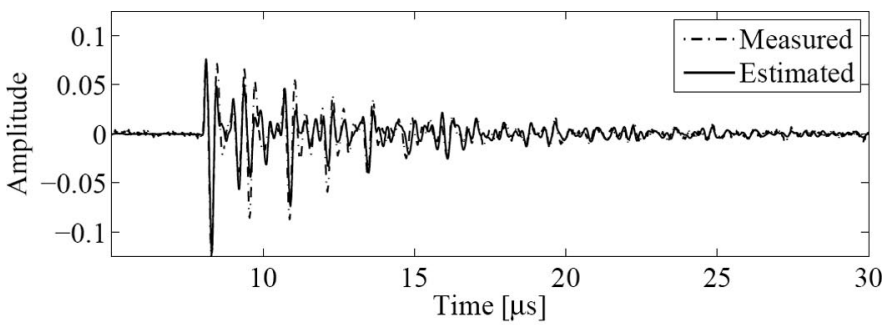

(a)

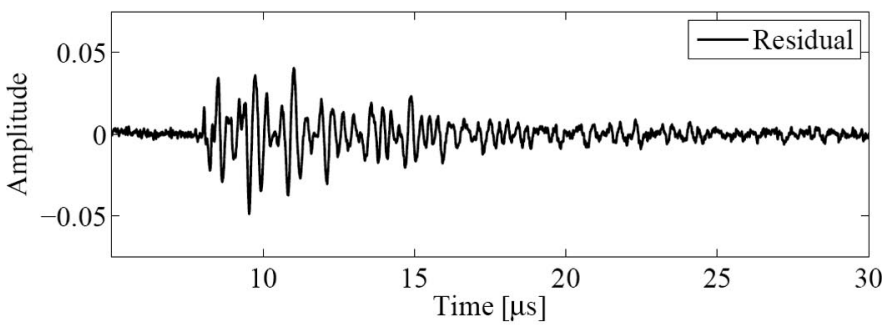

(b)

Fig. 11. (a) The measured output signal, $y_{\mathrm{m}}(t)$, from a thin 3-layered material and the estimated output signal, $y_{\mathrm{e}}(t),(\mathrm{b})$ the residual $e(t)=$ $y_{\mathrm{m}}(t)-y_{\mathrm{e}}(t)$.

ment situation the experimental setup and the surroundings influence the sound waves. The material properties are the ultrasonic attenuation and dispersion, and from the experimental setup we can have diffraction and misalignment effects. This implies that a complete physical (hard) model to describe all these effects is difficult to find and it might be too extensive and complicated for practical use. There is always the tradeoff between how much the model covers and how complicated the model gets. An approach is to just model some of the effects. Diffraction could, for example, be modeled physically by using the Lommel diffraction [31]. The attenuation is incorporated in the model, see Section II-C, but could be extended to a more complete model. A different approach to model the attenuation, dispersion, diffraction, and misalignment effects is to introduce empirical (soft) structures, in the form of finite impulse response (FIR) filters. This is done in [32], and the purpose of the soft structure is to cope with dynamics that the physical model is unable to handle. The benefit when including soft models is that a complete representation of the waveform is possible and there is nothing left in the residuals but measurement noise. This implies, for example, that signal separation of overlapping echoes is possible. The main drawback is that

table iII. Properties Extracted From the Parameters With Silicone and Epoxy as Bonding Material for Both the Pulse-Echo and the Through-Transmission Experiment.

\begin{tabular}{|c|c|c|c|}
\hline & Silicone $($ mean $\pm 2 \sigma)$ & Epoxy $($ mean $\pm 2 \sigma)$ & Glass pyrex $($ mean $\pm 2 \sigma)$ \\
\hline \multicolumn{4}{|c|}{ Pulse-echo measurement } \\
\hline$\hat{c}(\mathrm{~m} / \mathrm{s})$ & $980 \pm 0.1$ & $2337 \pm 0.3$ & $5797 \pm 0.1$ \\
\hline$\hat{\rho}\left(\mathrm{kg} / \mathrm{m}^{3}\right)$ & $1104 \pm 12.9$ & $982 \pm 1.9$ & $2370 \pm 5.1$ \\
\hline \multicolumn{4}{|c|}{ Through-transmission measurement } \\
\hline$\hat{c}(\mathrm{~m} / \mathrm{s})$ & $990 \pm 0.1$ & $2337 \pm 0.1$ & $5714 \pm 0.1$ \\
\hline$\hat{\rho}\left(\mathrm{kg} / \mathrm{m}^{3}\right)$ & $1171 \pm 20.0$ & $1157 \pm 11.3$ & $2220 \pm 18.2$ \\
\hline
\end{tabular}


the number of parameters increases and the model has a loss in simplicity and the estimation algorithm has a loss in speed and possibly numerical stability.

\section{B. Varying Measurement Conditions}

In the measurement situations where there are no possibilities to estimate the input signal from the same measurement as the output signal, the problem of different measurement conditions occurs. The problem is important in pulse-echo configuration, but it is even more important in through-transmission configuration because then there is always a need for a reference measurement, see Section II-E. Two different measurements on different media implies different experimental setups and different influence on the wave from the media. This means that the shape of the echoes might be different; that is, an echo traveling only through water might have a different appearance than an echo traveling through a solid material. A suggested solution is to add an FIR filter when estimating the input signal, where the FIR filter should cope with the new measurement conditions created when performing the new reference measurement. However, this is a more complicated method and might not be required when a good reference measurement could be conducted.

Another aspect to be aware of is that the hydrophone has a much better spatial resolution than the transducer, also implying that the received waveform has lower amplitude. The difference in spatial resolution implies that, for a not perfectly homogeneous layer, there might be some small differences in the estimated parameters.

\section{Performance Analysis of the Estimation Algorithm}

A possible extension to the work in this study, is to evaluate the performance of the estimator. This is done by comparing the minimum square error (MSE) to CramérRao lower bound (CRLB) that acts as a lower limit. The CRLB is given by the inverse of the Fisher information matrix (FIM) [26]

$$
\operatorname{FIM}_{\theta}=E\left\{\frac{\partial \varepsilon(\omega, \boldsymbol{\theta})}{\partial \boldsymbol{\theta}} \frac{\partial \varepsilon(\omega, \boldsymbol{\theta})}{\partial \boldsymbol{\theta}}\right\},
$$

where $\varepsilon(\omega, \boldsymbol{\theta})$ is found in (55) in Appendix A-C. Calculating the CRLB requires the gradients of the transfer function with respect the each parameter. Calculating these gradients requires an extensive effort.

The CRLB can, for example, be used to check where the limitations in the model and the estimation lie: the number of layers, the thickness of the layers, signal-tonoise conditions, environmental conditions, etc.

\section{CONCLUSIONS}

It has been shown with measurements using both pulseecho and through-transmission techniques that the para- metric model presented in this paper is able to reconstruct the ultrasonic signal waveform consisting of multiple overlapping echoes from within multi-layered structures by using the estimated parameter vector. The paper presents a system of linear equations that creates the opportunity to derive a desired model for an arbitrary number of layers with input and output above and/or under the sample. Given the model and its parameters, it is possible to extract material properties of the individual layers.

Measurements performed on 3-layered materials showed that the model is appropriate and could be used for estimating material properties.

\section{Appendix A}

\section{A. Newton Minimization}

The weighted error in (38) of the model is to be minimized and the NWLS estimator is found by minimizing the LS error function in (39). The iterative update of the parameter vector to find the minimum of (39) is

$$
\boldsymbol{\theta}^{\{i+1\}}=\boldsymbol{\theta}^{\{i\}}-\Delta \boldsymbol{\theta}^{\{i\}},
$$

where

$$
\Delta \boldsymbol{\theta}^{\{i\}}=\left.\left(\left.\frac{\partial^{2} J(\boldsymbol{\theta})}{\partial \boldsymbol{\theta} \partial \boldsymbol{\theta}^{T}}\right|_{\boldsymbol{\theta}=\boldsymbol{\theta}}\right)^{-1} \cdot \frac{\partial J(\boldsymbol{\theta})}{\partial \boldsymbol{\theta}}\right|_{\boldsymbol{\theta}=\boldsymbol{\theta}}\{i\}
$$

\section{B. Expressions for Jacobian and Hessian}

The Newton minimization requires the Jacobian (gradients) and the Hessian of $J(\boldsymbol{\theta})$ in (39). The Jacobian of the LS error and the Hessian can be expressed as

$$
\begin{gathered}
\frac{\partial J(\boldsymbol{\theta})}{\partial \boldsymbol{\theta}}=\frac{\partial\left\{\varepsilon^{H} \varepsilon\right\}}{\partial \boldsymbol{\theta}} \\
=2 \Re\left\{\frac{\partial \varepsilon}{\partial \boldsymbol{\theta}} \varepsilon\right\}, \\
\frac{\partial^{2} J(\boldsymbol{\theta})}{\partial \boldsymbol{\theta} \partial \boldsymbol{\theta}^{T}}=\frac{\partial^{2}\left\{\varepsilon^{H} \varepsilon\right\}}{\partial \boldsymbol{\theta} \partial \boldsymbol{\theta}^{T}} \\
=2 \Re\left\{\frac{\partial^{2} \varepsilon^{H}}{\partial \boldsymbol{\theta} \partial \boldsymbol{\theta}^{T}} \varepsilon\right\}+2 \Re\left\{\frac{\partial \varepsilon^{H}}{\partial \boldsymbol{\theta}} \frac{\partial \varepsilon}{\partial \boldsymbol{\theta}}\right\},
\end{gathered}
$$

where $\Re$ represents the real part.

\section{Gauss-Newton Method}

The approximative Hessian is in the Gauss-Newton method found by ignoring higher order derivatives, i.e., omitting the second-order derivative of $\varepsilon$, that is, the first term in the Hessian in (53). Hence, the update of the parameter vector in (51) is, in the Gauss-Newton linearization algorithm, given by 


$$
\Delta \boldsymbol{\theta}=\left(\Re\left\{\frac{\partial \varepsilon^{H}}{\partial \boldsymbol{\theta}} \frac{\partial \varepsilon}{\partial \boldsymbol{\theta}}\right\}\right)^{-1} \cdot \Re\left\{\frac{\partial \varepsilon^{H}}{\partial \boldsymbol{\theta}} \varepsilon\right\},
$$

using $\boldsymbol{\theta}=\boldsymbol{\theta}^{\{i\}}$. The gradient $\partial \varepsilon / \partial \boldsymbol{\theta}$ in the Gauss-Newton algorithm is

$$
\frac{\partial \varepsilon(\boldsymbol{\theta})}{\partial \boldsymbol{\theta}}=-\frac{\frac{\partial \mathrm{H}(\boldsymbol{\theta})}{\partial \boldsymbol{\theta}} \mathrm{U} \sigma_{e}(\boldsymbol{\theta})+(\mathrm{Y}-\mathrm{H}(\boldsymbol{\theta}) \mathrm{U}) \frac{\partial \sigma_{e}(\boldsymbol{\theta})}{\partial \boldsymbol{\theta}}}{\sigma_{e}^{2}(\boldsymbol{\theta})},
$$

where $\partial \mathrm{H}(\boldsymbol{\theta}) / \partial \boldsymbol{\theta}$ is the gradient of the transfer function. The variance of the error is given by

$$
\sigma_{e}^{2}(\omega, \boldsymbol{\theta})=\sigma_{y}^{2}(\omega)+\sigma_{u}^{2}(\omega)|\mathrm{H}(\omega, \boldsymbol{\theta})|^{2}
$$

where $\sigma_{u}^{2}(\omega)$ and $\sigma_{y}^{2}(\omega)$ are the estimated input and output noise variances, assumed to be uncorrelated. The input noise is colored by the magnitude of the transfer function. The gradient of this error is given by

$$
\frac{\partial \sigma_{e}(\boldsymbol{\theta})}{\partial \boldsymbol{\theta}}=\frac{\sigma_{u}^{2}\left(\frac{\partial \mathrm{H}^{*}(\boldsymbol{\theta})}{\partial \boldsymbol{\theta}} \mathrm{H}(\boldsymbol{\theta})+\mathrm{H}^{*}(\boldsymbol{\theta}) \frac{\partial \mathrm{H}(\boldsymbol{\theta})}{\partial \boldsymbol{\theta}}\right)}{2 \sigma_{e}(\omega, \boldsymbol{\theta})},
$$

where ${ }^{*}$ denotes complex conjugate. If there is no noise on the input signal, that is, $\sigma_{u}^{2}$ is equal to zero, then $\sigma_{e}^{2}(\boldsymbol{\theta})=\sigma_{y}^{2}$ and $\partial \sigma_{e}(\boldsymbol{\theta}) / \partial \boldsymbol{\theta}=0$. This simplifies the gradient of the weighted error in (55) to

$$
\frac{\partial \varepsilon(\boldsymbol{\theta})}{\partial \boldsymbol{\theta}}=-\frac{\frac{\partial \mathrm{H}(\boldsymbol{\theta})}{\partial \boldsymbol{\theta}} \mathrm{U}}{\sigma_{y}} .
$$

Nevertheless, the gradients of $\mathrm{H}(\boldsymbol{\theta})$ is needed, and calculating these gradients are essential for the algorithm. These gradients are used in (57) and finally the gradient of the error in (55) used to update the parameter vector $\boldsymbol{\theta}$ can be calculated. The gradients of $\mathrm{H}(\boldsymbol{\theta})$ are calculated with respect to every parameter in the parameter vector $\boldsymbol{\theta}$.

\section{REFERENCES}

[1] A. Fertner and A. Sjölund, "Comparison of various time delay estimation methods by computer simulation," IEEE Trans. Acoust. Speech Signal Process., vol. 34, no. 5, pp. 1329-1330, 1986.

[2] R. Freemantle, R. Challis, and J. White, "A z-transform technique for thin-layer reverberation cancellation applied to ultrasonic NDT of adhered structures," in IEE Colloq. Advanced Techniques for Collection and Interpretation of NDT Data, vol. 102, 1994, pp. 7/1-4.

[3] L. Wang, B. Xie, and S. Rokhlin, "Determination of embedded layer properties using adaptive time-frequency domain analysis," $J$. Acoust. Soc. Am., vol. 111, no. 6, pp. 2644-2653, 2002.

[4] C. Martin, J.-J. Meister, M. Arditi, and P.-A. Farine, "A novel homomorphic processing of ultrasonic echoes for layer thickness measurement," IEEE Trans. Signal Process., vol. 40, no. 7, pp. 1819$1825,1992$.

[5] V. Kinra and V. Iyer, "Ultrasonic measurement of the thickness, phase velocity, density or attenuation of a thin-viscoelastic plate. Part I: The forward problem," Ultrasonics, vol. 33, no. 2, pp. 95-109, 1995
[6] V. Kinra and V. Iyer, "Ultrasonic measurement of the thickness, phase velocity, density or attenuation of a thin-viscoelastic plate. Part II: The inverse problem," Ultrasonics, vol. 33, no. 2, pp. 111122, 1995.

[7] T. Kundu, "Complete acoustic microscopical analysis of multilayered specimens," J. Appl. Mech., vol. 59, no. 1, pp. 54-60, 1992.

[8] S. Rokhlin and W. Huang, "Ultrasonic wave interaction with a thin anisotropic layer between two anisotropic solids: Exact and asymptotic-boundary-condition methods," J. Acoust. Soc. Am., vol. 92, no. 3, pp. 1729-1742, 1992.

[9] J. Martinsson, J. E. Carlson, and J. Niemi, "Model-based phase velocity and attenuation estimation in wideband ultrasonic measurement systems," IEEE Trans. Ultrason. Ferroelectr. Freq. Control, vol. 54, no. 1, pp. 138-146, 2007.

[10] M. Feder and E. Weinstein, "Parameter estimation of superimposed signals using the EM algorithm," IEEE Trans. Acoust. Speech Signal Process., vol. 36, no. 4, pp. 477-489, 1988.

[11] R. Demirli and J. Saniie, "Model-based estimation of ultrasonic echoes. Part I: Analysis and algorithms," IEEE Trans. Ultrason. Ferroelectr. Freq. Control, vol. 48, no. 3, pp. 787-802, 2001.

[12] R. Demirli and J. Saniie, "Model-based estimation of ultrasonic echoes. Part II: Nondestructive evaluation applications," IEEE Trans. Ultrason. Ferroelectr. Freq. Control, vol. 48, no. 3, pp. 803811, 2001

[13] E. J. Chern, "An advanced ultrasonic system for defect image enhancement," in Proc. 1st US-Japan Symp. on Advances in NDT, 1996, pp. 133-137.

[14] E. P. Papadakis, "Online statistical process control with NDE and computers," in Proc. IEEE Int. Ultrason. Symp., Chicago, IL, 1988, pp. $523-527$.

15] J. S. Heyman, W. P. Winfree, F. R. Parker, D. M. Heath, and C. S. Welch, "Quantitative NDE applied to composites and metals," in Nondestructive Monitoring of Materials Properties Symp., Boston, MA, 1983, pp. 211-220.

[16] R. Mehrabian and N. G. Wadley, "Needs for process control in advanced processing of materials," J. Met., vol. 37, no. 2, pp. 51-58, 1985.

[17] D. E. Bray and R. K. Stanley, Nondestructive Evaluation: A Tool for Design, Manufacturing, and Service. New York: McGraw-Hill, 1989 .

[18] L. E. Kinsler, A. R. Frey, A. B. Coppens, and J. V. Sanders, Fundamentals of Acoustics, 3rd ed. New York: Wiley, 1982.

[19] G. Strang, Linear Algebra and its Applications, 3rd ed. San Diego: Harcourt Brace Jovanovich, 1986.

[20] R. Pintelon and J. Schoukens, System Identification: A Frequency Domain Approach, vol. 1. Piscataway, NJ: IEEE Press, 2001.

[21] L. M. Brekhovskikh, Waves in Layered Media, vol. 1. New York: Academic Press, 1960.

[22] F. Hägglund, J. Martinsson, and J. E. Carlson, "Flaw detection in layered media based on parametric modeling of overlapping ultrasonic echoes," in Proc. IEEE Int. Ultrason. Symp., 2006, pp. 136-139.

[23] F. Hägglund, J. Martinsson, J. E. Carlson, and C. Carlander, "Model-based characterization of thin layers using pulse-echo ultrasound," in Proc. Int. Cong. Ultrasonics, Vienna, Austria, Apr. 2007.

[24] F. Hägglund, Tools for Ultrasonic Characterization of Layered Media. Lic. Thesis, Luleå University of Technology, 2007.

[25] L. Ljung, System Identification: Theory for the User, 2nd ed. Englewood Cliffs: Prentice-Hall, 1987.

[26] S. M. Kay, Fundamentals of Statistical Signal Processing: Estimation Theory, vol. 1. Englewood Cliffs: Prentice Hall, 1993.

[27] R. Fletcher, Practical Methods of Optimization, 2nd ed. New York: Wiley, 1991.

[28] P. Guillaume and R. Pintelon, "A Gauss-Newton-like optimization algorithm for "weighted" nonlinear least-squares problems," IEEE Trans. Signal Process., vol. 44, no. 9, pp. 2222-2228, 1996.

[29] L. Peirlinckx, R. Pintelon, and L. P. van Biesen, "Identification of parametric models for ultrasonic wave propagation in the presence of absorption and dispersion," IEEE Trans. Ultrason. Ferroelectr. Freq. Control, vol. 40, pp. 302-312, Jul. 1993.

[30] C. Graciet and B. Hosten, "Simultaneous measurement of speed, attenuation, thickness and density with reflected ultrasonic waves in plates," in Proc. IEEE Int. Ultrason. Symp., 1994, pp. 1219-1222.

[31] P. Rogers and A. V. Buren, "An exact expression for the Lommel diffraction correction integral," J. Acoust. Soc. Am., vol. 55, pp. 724-728, Apr. 1974. 
[32] J. Martinsson, F. Hägglund, and J. E. Carlson, "Complete postseparation of overlapping ultrasonic signals by combining hard and soft modeling," Ultrasonics, vol. 48, no. 5, pp. 427-443, 2008.

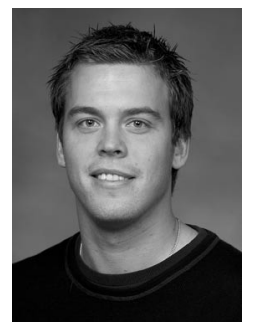

Fredrik Hägglund was born in Örnsköldsvik, Sweden, in 1980. He received his M.Sc. degree in electrical engineering in 2005, and a Licentiate degree in electrical engineering in 2007, from Luleå University of Technology, Sweden. He is currently pursuing a Ph.D. degree in electrical engineering at Luleå University of Technology. His research includes ultrasonic measurement technology and signal processing.

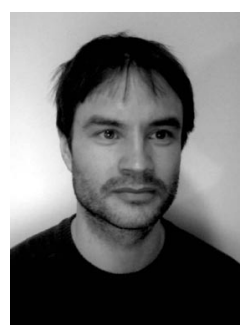

Jesper Martinsson was born in Gällivare, Sweden, in 1978. He received his M.Sc. degree in computer science and engineering in 2004, and a Ph.D degree in electrical engineering in 2008, from Luleå University of Technology, Sweden. He currently works with mining $\mathrm{R} \& \mathrm{D}$ at LKAB. His primary research interests include acoustics, signal processing, and measurement technology.

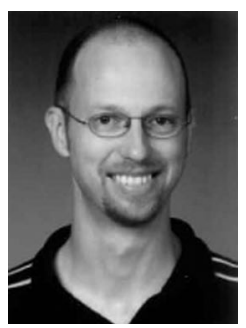

Johan E. Carlson was born 1973 in Umeå, Sweden. He received his M.Sc. degree in computer science and engineering and his Ph.D. degree in electrical engineering at Luleå University of Technology, Sweden, in 1998 and 2002, respectively. In 2006, he was appointed Associate Professor and received the young researcher award from the Royal Skyttean Society. He currently holds a position as senior lecturer at the Dept. of Computer Science and Electrical Engineering at Luleå University of Technology, Sweden. His research interests include ultrasonic measurement technology, signal processing, and multivariate data analysis. 